\title{
XII. Aus der sogen. Manuskripten-Sammlung des kgl. allgemeinen Reichsarchivs.
}

\author{
Von \\ Dr. Carl Schaefer, Reichsarchivpraktikant in München.
}

Bis zum Jahre 1874 bildete die sogen. Manuskripten-Sammlung des k. allgem. Reichsarchivs eine in sich geschlossene Sammlung der verschiedenartigsten schriftstellerischen Erzeugnisse aus älterer und neuerer Zeit, welche sämmtlich aus den mannigfachsten Veranlassungen, wie durch Ankauf, Schenkung, Vermächtniss, Staatsverwaltungsakte, behördlichen Austausch u. dgl., dem Reichsarchive als einzelne Bestandtheile zugefallen warẹn, zufolge ihrer besonderen Herkunft an einem gesonderten Orte - in der zweiten Abtheilung der Amtsbibliothek - aufbewahrt wurden und dort gleichsam ein Archiv im A rchive bildeten.

Die Bezeichnung „Manuskripten-Sammlung" deckte zur Zeit ihrer Entstehung vermuthlich sich mit deren Inhalt. In späteren Jahren aber, als man die anfallenden Stücke, ohne eine zeitraubende Ausscheidung der einzelnen Bestandtheile erst vornehmen zu wollen, unmittelbar der einmal hiefür geschaffenen Sammlung anreihte, ist Manches hineingekommen, was mit dem Gegenstande, den wir mit dem Worte „Manuskript“ heutzutage bezeichnen, nur mehr die äussere Erscheinungsform gemein hatte. Mit dem durch die Entwickelung der Landeshoheit Hand in Hand gehenden Zerfall der Autonomie der Reichsstadte, mit der im Jahre 1799 eintretenden Reorganisation der Landesarchive, ferner mit der Säkularisirung der Reichsstifte und Klöster vom Jahre 1803 an, gelangte eine Unmasse von geschriebenem und gedrucktem literarischen Material aus den städtischen und klösterlichen Archiven, Bibliotheken und Schulen, sowie aus den Staatsregistraturen und geheimen Kanzleien in den Alleinbesitz der 
Landesregierung und ihrer Archive. Auch dem k. geheim. Landes-, jetzt $k$. allgem. Reichsarchive, wurde auf diese Weise ein überaus grosser Schatz des reichhaltigsten Akten- und Urkundenstoffes mit einer Menge handschriftlicher Aufzeichnungen zugeführt. Fin im Reichsarchive vorhandenes Blätterverzeichniss - gefertigt von dem ehemaligen Reichsarchivfunktionär Dr. Roth, später ergänzt durch Reichsarchivrath Primbs - gibt Aufschluss über den bis zum Jahre 1874 auf nahezu 2500 Einzelnummern herangewachsenen Bestand der Manuskripten-Sammlung.

Schon das aus Stiften und Klöstern dem k. Reichsarchive an Chroniken, Stifts- und Todtenbüchern, an universalgeschichtlichen Kalendarien, Tagebüchern, Verzeichnissen, Biographien, Legenden, Liedern, Hymnen, Lob- und Festgedichten, Schwänken und Festspielen Angefallene - nebst einer kleineren Sammlung Breviarien, Psalterien, Missalen, Choralbüchern und Glossarien aus dem 14. und 15. Jahr'hundert, zum Theile aus Freising stammend, - würde genügt haben, ein Kulturbild aus den letzten drei Jahrhunderten zu entrollen und den ungemein grossen Fleiss, die wissenschaftliche und schriftstellerische Thätigkeit der Klostergeistlichkeit näher zu kennzeichnen.

A ber auch eine nicht minder grosse Zahl von „Städte-Chroniken“ aus den Reichsstädten Augsburg, Nürnberg, Salzburg, Regensburg (über Regensburg allein 53), fast alle aus dem 16. und 17. Jahrhundert stammend, mehr oder weniger vollständig, sind auf diese Weise Eigenthum des Landesarchivs und Bestandtheil der Manuskripten-Sammlung geworden.

Es gelangte ferner, meist als "Nachlass" zufolge Ankaufs, oder Schenkung erworben, in die Manuskripten-Sammlung eine überaus grosse Zahl handschriftlicher Aufzeichnungen über die Gesammtgeschichte Bayerns und seines Fürstenhauses, deren Erforschung in genealogischer, heraldischer, numismatischer, topographischer und kulturhistorischer Richtung sich bayerische Archivare und Kanzler behufs Abfassung von Geschichtswerken zur Aufgabe gestellt hatten.

Nicht minder waren die Rechtswissenschaft und das Schulwesen - erstere durch eine Anzahl Kommentare, Kompendien, Dissertationen, Glossarien von den ehemaligen Universitäten Ingolstadt und Landshut, letzteres durch eine Reihe historisch-philosophischer Abhandlungen, Uebersetzungen, Reden, Geschichtlicher Tabellarien und Annalen, Beschreibungen, Lektionen und Episteln u. dergl. in der Manuskripten-Sammlung vertreten. 
Naturgeschichtliche, wie medizinische Abhandlungen aus frülieren Jahrhunderten, Konvolute ungeordneter Schriften- und Brief-Sammlungen von geistlicher und weltlicher Hand, endlich eine spezialgeschichtlich nicht uninteressante Sammlung von amtlichen Registern, Amts-, Saal- und Kopialbüchern aus den-ehemaligen herzoglichen, kurfürstlichen und pfalzgräflichen Archivregistraturen und geheimen Kanzleien bildeten den Abschluss des Ganzen.

Dies in Kürze der Bestand der sogen. Manuskripten-Sammlung bis zum Jahre 1874 als einer in sich geschlossenen Sammlung im Reichsarchiv. War auch Manches darin unvollendet oder ab- und zusammengeschrieben, Vieles durch späteren Druck bereits bekaunt gegeben, so war doch nicht zu verkennen, dass die Sammlung noch werthvolle Einzelheiten aus älterer und neuerer Zeit in Fülle in sich barg, und es war gewiss nicht ohne innere Berechtigung, wenn man sie als ein schätzbares Unikum betrachtete; ohne sie in ibren einzelnen, viel zerstreuten Bestandtheilen näher kennen zu lernen. Wem aber kam das zu Nutzen? Fast Niemand. Aktenmåssig lag hier begraben, was Fleiss und Jahre lange Forschung mühsam und als Stückwerk zu Pergament oder zu Papier gebracht. Die lokale Absonderung des Ganzen, sowie der Mangel jeglicher Eintheilung erschwerte die Benützung des Vorhandenen noch mehr.

Sollte die grosse Sammlung für Staat und Wissenschaft Früchte bringen, so musste sie bis zum Grunde gesichtet und klar gelegt, sodann aber jeder Bestandtheil dorthin gebracht werden, wohin er seinem Jnhalte nach gehörte, dorthin wo man Aelınliches vermuthete und daher auch allein aufsuchte. Also die Schriftstiicke, Hefte und Bände archivalischer Natur waren zu den verwandten Gruppen und Reihen in den Archiven einzutheilen, das Uebrige, insbesondere was Schriftstellerfleiss lediglich zu literarischen Zwecken erforscht und niedergeschrieben hatte, war der Staatsbibliothek zu übergeben, in welcher es am ehesten gesucht wurde und auch der Benützung leichter und bequemer zugänglich war. Ob schon gedruckt oder noch Handschrift - war ja unwesentlich, entscheidend die Entstehung und der Zweck, welcher den Verfasser leitete. Die Grundsätze solcher Ausscheidung alten, rein literarischen Stoffes, dessen noch genug Unbekanntes und Unbenutztes in vielen Archiven lagert, sind schon früher in der Archivalischen Zeitschrift - insbesondere im ersten Bande Seite 53 bis 55 - auseinandergesetzt.

Demgemäss wurden in den Jahren 1874 bis 1881, nachdem 
die ganze Masse der sogen. Manuskriptensammlung bis in 's Einzelne hin durchgearbeitet, verzeichnet und die ministerielle Genehmigung eingeholt war, von Dr. Geib - damals Reichsarchivassessor, jetzt Kreisarchivar von Oberbayern - unter Leitung des Reichsarchivdirektors nicht weniger als 900 Einzelnummern ausgeschieden und der k. Hof- und Staatsbibliothek in 12 Ablieferungen überreicht, während man 379 Einzelnummern im Reichsarchive selbst an geeigneter Stelle einreihte, wodurch insbesondere die Bestände der Reichsstädte, Klöster, Stifte, Hochstifte und Domkapitel, ferner der Reichs- und Kreissachen, der Gerichtsliteralien und des Adelsselekts eine namhafte Vermehrung erhielten. Ausserdem wurden an die acht bayerischen Provinzialarchive 119 Nummern der ManuskriptenSammlung extradirt, von denen die meisten Stücke an die Kreisarchive in Nürnberg und München gelangten. Auch dem Archivalienaustrusch der mit Baden gepflogen wurde, kam eine Nummer zu Gute, eine andere wurde an das k. geheim. Staatsarchiv extradirt. Ein sehr stattlicher Rest, der nun noch zurückblieb, eignete sich weder zur Eintheilung unter die Archivalien, noch zur Einreihung in die-Staatsbibliothek, liess sich dagegen zu drei Sondergruppen hervorbilden. Die erste umfasst die vielen Saal- und Lehenbücher von 1277 an, spätere Kammer- und Amtsbücher, in denen sich selbstverständlich ebenso wie in den zahlreichen alten Kopialbüchern auch Urkunden, Hand werksordnungen, Geschlechtsnachweiso, fürstliche Heirathsverhandlungen und dergleichen finden. Die zweite Gruppe besteht ans Repertorien-Arbeiten früherer Archivare, auch betitelt Register, Registraturen, Rezessbüücher, Beschreibungen, Renner und Indices. Aus beiden Gruppen theilen wir später vielleicht noch Mehreres mit, da aus der erstern sich anziehende und wichtige Beiträge für die Kulturgeschichte, insbesondere deren volkswirthschaftliche Seite, ziehen lassen, die Schriftwerke der alten Archivare aber nicht wenig ergeben für die Geschichte unseres Archivwesens; insbesondere des bayerischen, für welche durch des jetzigen Reichsarchivassessors Neudegger Arbeiten bereits schöne Ergebnisse gewonnen sind. Endlich die dritte Gruppe setzte sich aus dem Urkundenstoff zusammen, wie er in dem umfangreichen literarischen Nachlass früherer Archivare und anderer Beamten gesammelt ist im Interesse bayerischer Geschichte, Topographie, Genealogie und Heraldik. In dieser letzten Gruppe befindet sich noch, ausser einer „Cronikh des Landt Kărndtas" v. J. 1560 von Michael Arroden, 
die von Archivar Lieb in 24 Quartbănden rerfasste, gewöhnlich mit dem Namen „Excerpta genealogica et heraldica" bezeichnete Sammlung genealugischer Notizen über sämmtliche bayerische Adelsgeschlechter mit beigesetzten heraldischen Federzeichnungen - das Ganze eine Fundgrube für Genealogen.

Sodann gleichfalls von $\mathrm{Li}$ eb ist vorhanden ein zum Hund'schen Stammenbuch verfertigter "Index der Örther, Ämbter vnd Authorn" und „Additiones" zu den Adnotationen jenes Stammenbuchs.

Aus Lori's Nachlass sind hervorzuheben dessen umfangreiche Vorarbeiten zu einer „bayerischen Kirchengeschichte“ und zu einer „Geschichte des bayerischen Fürstenhauses", bestehend in einer überaus grossen Zahl von Urkundenkopien, ferner dessen 10 Bände starkes „bayerisches Urkundenregister" vom 6. bis 18. Jahrhundert, nebst einigen geschichtlichen Aufzeichnungen über den Lechrain. Ausser diesen sind noch: Jos. Anton Aettenkhover's „Continuatio ad Liebum", gleichfalls 24 Bände umfassend, ein Index über sämmtliche Lieb'sche genealogische Bücher, und ein Diplomatarium historicun Brandenburgense" von Archivar Schütz in 7 Cartons zu nennen, welchen sich eine ähnliche A rbeit „die Brandenburgischen Hausverträge", vermuthlich von Archivar Spiess zusammengestellt, anreiht.

Aus der Menge der handschriftlichen Werke, die aus der Manuskripten-Sammlung des Reichsarchivs an die Hof- und Staatsbibliothek im selben Gebäude abgegeben sind, heben wir jetzt eine Anzahl hervor, damit sie doch einigermassen bekannter werden und Geschichtsforscher und Liebhaber Gelegenheit erhalten, sich an ihnen zu erholen.

$$
\text { Kalendarien und Tageblicher. }
$$

Meichelbeck, Karl. Diaria ejusdem ab anno 1692-1734. 3 Konvolute $4^{0}$. - Nucleus memorabiliorum gestorum ejusdem patris. 1 kl. Konv. $8^{0}$.

Benediktbeuren, Kloster. Kalender vom Jahre 1750 mit tagebuchartigen Einzeichnungen.

- Verzeichniss der Klosterbesucher v. J. 1791.

- Diarien aber die taglichen Gaste und sonstige Begebenheiten im Kloster v. J. 1785-1791. J Konv. 8 Lib. $4^{0}$.

A 1 to munster, Kloster. Tagebuch v. J. 1691-1698. Continuatio rerum notabilium in monasterio St. Altonis.

Elchingen, Kloster. Tagebacher des Pater Joseph Linder und Anderen v. J. $1700 \rightarrow 1725$ und 1780.1 Bd. $4^{0}$.

Ottobeuren, Kloster. Miscellanea Ottoburans.

Tegernsee, Kloster. Tagebuch des Abtes v. J. 1796-1811. 1 Fasz, $4^{0}$. 
Aventin, Johann. Hand- und Hauskalender mit kritischen Bemerkungen von Anton Nagel. 1 Fasz. $4^{0}$.

Nagel, Anton. Tagebücher mit Autobiographie v. J. 1803-1810.

Dingolfing, Kloster. Diarium ïber die Ereignisse in den Jahren 1742/43 wăhrend des bayerisch österreich. Krieges von Pater Antigonus Schaur. $1 \mathrm{Bd} .4^{0} .81 \mathrm{~S}$.

Schwabach, Stadt. Hemerologium Brandenburgicum, oder Tagregister des Hauses Brandenburg v. J. 1748. $1 \mathrm{Bd}$. fol. $56 \mathrm{Bl}$. mit gedrucktem Titelblatt.

Oefelin. Literarischer Nachlass: Calendarium historicunn Bavaro-Palatinum de 1180-1629. 2 Lib. fol. u. $4^{0}$.

- Diarium des Konzils zu Konstanz in den Jahıren 1414-1418. 1 Bd. fol. 17. Jahrhundert.

Biographien und Reisebeschreibungen.

Friedrich II., König von Preussen. Person und Lebensordnung.

Descrïtio peregrinationis a Gallis, Belgis aliis religiosis Hiero. sol y ma m factae annis 1542 et 1543 . $1 \mathrm{Lib} .4^{0}$.

W u rmer, Jakob, der Aeltere. Beschreibung seiner Reise von Strassburg nach Jeru sal em i. J. 1560. 1 Tom. $4^{0}$. Goldschn. Ledereinbd. 1564.

Dohna, Frhr. Christophor v. Ruisen von Heidelberg nach Italien etc. in den Jahren 1600-1613. 1 Lib. $4^{0}$.

Hainh ofer, Philipp, Patriziers zu Augsburg Reise zu Herzog Philipp von Pommern v. J. 1617 mit Abbildungen. 1 Heft fol.

Gersten berg, Marc. v., sächsischer Kanzler. 2 Hefte fol.

Stein, Mich., canonic. Rebdorfensis. Vita 1784. 1 Lib. fol.

Keusslin, Albert, abbat. in monasterio Ottoburani. Vita. 18. Jahrh. $1 \mathrm{Lib}$. fol. Altmann, Bischof von Passau. Lebensbeschreibung des Stifters des Klosters St. Nicolai. 3 Lib.

Meissel. Notizen zum Leben des Conrad Celtes. 1 Fasz.

Delling, Joh. Leben Kaiser Ludwig des Frommen. 1 Fasz.

- Vita sancti Severini monachi. 1 Fasc.

Pappenheim, Alex Graf v. Lebens- und Reisebeschreibung in's gelobte Land, dem Abte von Kempten gewidmet und ubergeben zur Aufbewahrung im Stifte 1612. 1 Lib. $120 \mathrm{Bl}$. fol.

Lori, literarischer Nachlass. Lebensbeschreibung des Joh. Bapt. Fickler.

Franziskanerorden, Munchen. Beschreibung der Reise einer Deputation des Ordens von München nach Murcia in Spanien 1756. 1 Bd. $16^{\circ}$.

Rudhart, G. Th. Dr. Lebensbeschreibungen der in der Ruhmeshalle aufgestellten berahmten Munner Bayerns. 1 Conv. fol 1854.

Moritz, Joseph, Pater. Beschreibung einer Reise durch Oberbayern 1798, mit Bezugnahme auf Klöster-Archive, -Bibliotheken und deren Schätze.

Johanns und Albrechts Markgrafen von Brandenburg Beschreibung der Pilgerfahrt zum heiligen Grab im Jahre 1435. Abschrift. 14 Bg. 1 Fasz. 
Kataloge und Tabellen.

Bücherkatalog der Senatsbibliothek in curia (Regensburg) nebst Auf zeichnungen über seltene Bücher. 17. Jahrh. 1 Konv. fol.

Series abbatum cellæ $\mathrm{St}$. Galli in Helvetia. 16. Jahrh. 1 Lib. $4^{0}$.

Cat a log u s manuscriptorum bibliothecæ S. Bartholomæi Franc of urt. exclesi $x$

- sacrarum reliquiarum etc. sacelli in monasterio U t tenpurchae. 1598.

1 Bd. $4^{0}$.

Katalog der dem Jesuitencolleg zu Löwen von Bischof Torrentius zu Antwerpen hinterlassenen Münzen u. Gemmen. 18. Jahrh. 1 Bd. fol.

Synchronistische Tabellen der Päpste, Kaiser, bayerischen Herzöge, Bischöfe v. Eichstädt von G. Roth. 1799. 1 Lib. $4^{0}$.

Chronologische Geschichtstabellen v. J. 1500-1596. 1 Bd. fol.

Verzeichniss der Bischöfe, Weihbischöfe, Generalvikare von Augs burg, Freising, Regensburg bis zum Jahre 1750. 1 Bd. fol.

Katalog aller Erzbischőfe von Salaburg bis 1560 . 16. Jahrh. 1 Lib. $4^{0}$.

Catalog us manuscriptorum monasterii Benedictoburani. 1735̄. $1 \mathrm{Heft}$ fol.

- manuscriptorum monasterii Ottoburani. 18. Jahrh. 1 Heft. $4^{0}$.

- abbatum monasterii St. Petri Salisburgens. 1664. Abschrift. 18. Jahrh. 1 Bd. fol.

Verzeichniss der Burggrafen von Nürnberg. 17. Jahrh. 1 Lib. fol.

Kata log der Klosterbibliothek zu RamBau. 12 Hefte. fol.

- der Manuskripte des Klosters Reis ach v. P. Coelestin Angelsprugger. 1769. 1 Bd. fol.

General-Index zu den gedruckten Deduktionen der Btadt Linda u uber deren Streitigkeiten mit dem Stift v. J. R. Wegelin. 1753. 1 Bd. fol.

\section{Briefwechsel.}

Benedictbeuren, Kloster. Briefe an die Aebte des Klosters. 1 Fasz. fol. Nage l, Anton. Wissenschaftlich-historische Privatkorrespondenz v. J. 1790 bis 1812. 2 Fasz.

Lipowsky, F. J. Original-Korrespondenz über Agnes Bernauerin. 1 Konv. fol. Attel, Kloster. Briefe über Aufhebung der Klöster Kladrau und Kremsmünster. $1786-1788$.

1594-1659. Briefe betreffend den 30jahrigen Krieg. 1 Folioband.

Merer, Hans. Briefwechsel mit Stefian Fugger zu Regensburg. 5 Bde. fol. 1583-1595.

A ettenkhofer, Jos. Ant. Korrespondenz mit Verleger Montag in Regensburg und verschiedenen Gelebrten. 1765-1768. 1 Fasz. 36 Prod.

Moritz, Joseph, Pater. Korrespondenz mit P. Braun, Nagel, J. E. Haindl und Andern. 4. Fasz.

Birzele, Ulrich, Neuburg. Korrespondenz mit Goswin Midder. 1790. 4 Fasz.

Günthner, Sebastian. Korrespondenz über seine Thatigkeit als Schriftsteller und Akademiker. 1818. 1 Konv fol.

Ortskunde.

Hucker, Joh. Geographisch-historische Beschreibung aller Lander- und Bisthomer in der Oberpfalz. 1 Fasz. fol. $358 \mathrm{~S}$. 
Steininger. Histor.topographische Beschreibung des Marktes Geisenhausen. I Fas\%. 4".

Lipowsky, Anton. Literarischer Nachlass. Karten und Pläne von Bayern etc. 1 Fasz. fol.

Lang, C. H. v. Collectanea zur alten (ieographie und den deutschen Rechtsund Alterthümern. 1 Fasz. 80. 25 Prod.

Morit\%, Jos. Literarischer Nachlass. Geographisch-historische Arbeiten über Elchingen, Lauingen, Gunzburg und Druipburg. 1 Fasz. fol.

-- Literar. Nacblass. Notizen tiber die altesten Orte um das Kloster Kastel. 1 Fasz. fol.

- Literar. Nachlass. Alphabet. topographisch-genealogische Register mit Anmerkungen. 1 Fasz. fol.

A pian, Phil. Topographie von Bayern. Originalhandschrift mit Handzeichnungen und Holzschnitten. 4 Hefte fol. $390 \mathrm{~s}$.

Burgholzer, Jos. Topographische Karte der Mönchskultur in Bayern v. J. 400-1300. $1 \mathrm{Bl}$. Federzeichnung.

\section{Kriegswissenschaft.}

Faul haber, Joh. Architectura militaris v. J. 1636. 1 tom. gr. fol. nebst Rissen. Furtenbach, Jos. Anleitung zur Erbauung von Schanzen und sonstigen Kriegsbanten v. J. 1664 . 1 Heft $4^{0}$.

Orfeo Galliani, Colonello. Libro secondo delle stratageme militari et inventione usate. 17. Jahrh. 1 Heft fol.

Neudecker. Literar. Nachlass. Russisches Exerzier-Reglement v. J. 1730. 1 Heft fol.

Danner. Literar. Nachlass. Der Rückzug der Franzosen in Jahre 1812. 1 Heft fol.

- Zeichnungen und Plane eines Distanzmessers für Mörser, über Geschütz. aufstellung in Kasematten und Anderes. $4 \mathrm{Bl}$. in einer Rolle.

\section{Urkundenlehre.}

Lang, C. H. v. Literar. Nachlass. Auszüge aus Khevenhiller und Fejer's Codex diplom. Hungariæ. 1 Fagz. $4^{0}$.

- Literar. Nachlass. Collectanea diplomatica betr. Archive, den nouveau traité de diplomatique, Chronologie, Siegel, Kaiserregesten. 1 Konv. $4^{0}$.

Morit $z$, Jos. Materislien zu einer Kritik des in den Monum. Boic. X. $381 \mathrm{ff}$. abgedruckten Saalbuchs des Klosters Scheurn. 1 Fasz. fol.

- Ueber tironische Noten. 1 Konv. fol.

- Tabulæ chronologico-diplomatico-historiæ negotiorum sub regimine Lotharii III., Conradi II., Friderici I. diplomatarium de 1123-1109. 4 Fasz. 1 Konv.

- Monumenta Ratisponensia, Urkundenauszuge und Notizen über das Hoch. stift Regensburg und die dortigen Klöster. I Konv. fol.

- Codex diplomaticus comitum de Sulzbach. 1 Fasz. fol.

- Codex traditionum Ensdorfensium mit Vorrede und Noten. Druck. manuskript. 1 Fasz. fol. 
Naturwissenschaft und Medizin:

Lullii, Raimundi Maioricani Liber alchymisticus. 16. Jahrh. 4". $365 \mathrm{~S}$. Ortolf, Maister. Arzneibuch, lat. v. J. 1470. 1 tom. fol., rother Ledereinband. Pflaundorffer, Heinrich. Arzneibuch v. J. 1450. 1 tom. in Holzdeckel. fol. Andechs, Kloster. Tractatus diversi de rebus naturalibus. Papierhandschrift mit Pergament durchschossen. 15. Jahrh. 1 tom. fol. $263 \mathrm{Bl}$.

Freising, Domkapitel. Libri physicorum. 15. Jahrh. 1 tom. fol.

Mundinus et Dynus de Florentia. Tractatus diversi de medicina. 15. Jahrh. 1 tom. fol.

Africanus, C. monach. Montis Casinensis. Liber de scientia rerum natural. et Magistri Gentilis de Fulgineo med. doctoris recepta. 1478. 1 tom. $4^{0}$.

-- Historia naturalis. Papierhandschrift des 15. Jahrhunderts. 1 tom. $4^{\circ}$. Andech8, Kloster. Præcepta, de remediis in variis morbis. 2 Sammelbände. 15. Jahrh. $4^{0}$.

Burgholzer. Joseph. Excerpte über die Bevoblkerungs- und Krankheits verhaltnisse der Stadt München. 1 Conv. $4^{0}$.

- Abbildung und Beschreibung von Giftpflanzen mit 16 colorirten Tafeln. 1 Fasz. fol.

\section{J urisprudenz.}

Freising, Domkapitel. Glossarium über das corp. juris. 15. Jahrh. Papierhandschrift. 1 tom. gr. fol.

Diemair, J., Syndikus zu Regensburg. Collectanea juridica etc. v. J. 1570 bis $1590.1 \mathrm{Bd}$. fol.

- Summarischer Auszug aus der bayerischen Landsordnung. 1648. $1 \mathrm{Bd} .4^{0}$.

Delling, Joh. v. Bayern's Rechtswesen. 5 Easz. 1 Konv.

- Staatsrechtswesen und Geschichte von Frankreich. 1 Konv.

- Münchener Stadtrecht v. 1347 nebst Gantordnung. 4 Fasz. fol. 1 Konv.

Unertl. Literarischer Nachlass. Ad Hieroglyphicum oder Codex bavaric: Von den ersten Ministris bei Hof, von den Gesandten und Generalen 3 Bde. $4^{0}$. Led. Goldschn.

- Europäische Staatsakten aus den Jahren 1594-1659, einzelne Akten. stücke aber den 30jahrigen Krieg enthaltend. $1 \mathrm{Bd}$. Fol.

Moritz, Joseph. Literarischer Nachlass. Herrschaftsgerichte in Bayern. 1 Fasz.

Ehinger, Dr. Juristischer Nachlass. 17. Jahrh. 1 Foliobd.

- Norma practicandí in Styria, steyermärkische Civilprozessordnung. 1 Bd. fol.

Freyberg, Frhr. v. Das Rechtsbuch der Stadt Memmingen de 1396. Abschrift. 1 Fasz. fol.

Narmi, Dr. Fabius v. Gutachten in lehensrechtlichen Fragen. Ingolstadt 16. Jahrh. Original mit Siegel. 1 Lib. fol. $91 \mathrm{Bl}$.

Mortoigne, Moses van. Staatspolitische Berichte in hollandischer und französischer Sprache v. J. 1688-1689. Originalhandschriften an Prinz Wilhelm von Oranien. $1 \mathrm{Bd} .4^{0} 371 \mathrm{~S}$.

Mortoigne, Moses van. Bergordnung und Bergrecht der Stadt Schemnitz in Ungarn. 17. Jahrh. 1 Bd. fol. 
Aus der sng. Manuskripten-Sammlung des k. allgem. Reichsarchivs. 235

M or it z, Joseph. Literarischer Nachlass. Alhaudlung ülser das muhamedanische bezw, türkische Recht.

- Notizen über das bayerische und auswärtige Armenwesen.

- Alhandlung über Freiheit des Handelsverkehrs.

Lori, Joh. Georg v. Anmerkungen über v. Moser's bayer. Staatsrecht. 1 Fasz. fol.

\section{Theologie.}

Kegensburg, Stadt. Annus columbinus, taglicher Muttergotteskalender 1704. 1 Bd. kl. fol.

- Vita sive legenda cum miraculis douninat Katherinæ. 15. Jahrh. 1 Lib. 4".

- Gregorii papæe regula pastoralis et liber dialogorum. Pengamentcolex des 10. Jahrh. aus Kloster Priffling. 1 Bd. kl. fol.

- Lectiones et epistolæ in diebus dominicis et festivis legendæ. Pergamenthandschrift. 14. Jahrh. Mit Initialen. $1 \mathrm{Bd}$. fol.

Franciscus, abbas Pollingensis. Dominicale ab a: 1735-1744. Predigten. 1 Bd. 4".

Ottenh ofer, Erhard. Rector parochial. eccles. in Neuching. Tractatus ab a: $1428-1434$.

Holqu ot, Robert. Questiones sive lectura super quatuor libros sententiarum. Pergamentcodex. 14. Jahrh. 1 tom. gr. $4^{0}$. aus Freising.

Dinkelspühel, Nicolai de. Sermones pars I. 1432. 1 tom fol. aus Freising. Witigo, episc. Missniensis. Miracula Bennonis. Pergamenthandschrift. 14. bis 15. Jahrh.

Tegernsee, Kloster. Passio St. Quirini. 16. Jahrh. 1 Lib. fol.

Ka i sershei m, Kloster. Psalterium. Pergamentcodex 11. u. 12. Jahrh. 1 tom. $4^{0}$. - Psalterium. Pergamentcodex dés 14. Jahrh. 1 tom. $4^{0}$.

Mallersdorf, Kloster. Missale Romanum. Pergamentcodex des 14. Jabrh. 1 tom. $4^{4}$.

Paering, Kloster. Homiliæ et sermones. Pergamentcodex. 1 tom. 4". Andechs, Kloster. Tractatus theologico-mysticus. 15. Jahrh. 1 tom. 4".

Cra m in er, Anton. Apostolische Glaubenshelden sus den Churbäusern Bayerns und der Pfalz. 17831 Bd. $4^{0}$.

- Ratisbona sancta et sacra. 1764-1782.

- Frisinga sancta. 1772.1 Bd. $4^{0}$.

- Oettinga sacra. $1780.1 \mathrm{Bd} .4^{0}$.

- Geschichte der Heiligen und Seligen des Bisthums Augsburg. 1784. I Bd. $4^{0}$.

- Salisburgum sanctum. 1771. 1 Bd. $4^{0}$.

Mariastein, Kloster. Gebete und geistliche Betrachtungen. 1j. Jahrb. 1 Bd. $16^{\prime}$.

Il 1 m ü nst e r, Kloster. Legenda de beato Arsacio episc. Pergamenthandschrift. 16. Jahrh. 1 Lib. fol.

Erf urt, Kloster. Tractatus theologici. 1398. 1 Bd. $4^{u}$ aus St. Peter zu Neuburg. Neuburg, Kloster. Sermones de 1393-1413. Papierhandschrift. 3 tom fol. Ne u burg, Kloster St. Peter. Liber pro informatione sacerdotum 1392. Papierhandschrift. 1 Bd. fol. 
Le u te n bach, Kloster St. Johann. Missale. Pergamenthandselırift. 15. Jahrh. 1 tom. 4".

- Breviarium. Pergamenthandschrift 15. Jahrh. Mit Kircheugesäingen und Noten. 1 tom. kl. $\mathbf{4}^{\mathrm{u}}$.

- Breviarium. Papierhandschrift. 14. Jahrh. 1 toin. 4".

Monasterium Mellicens. Breviarium ceremoniale de 1418-1487. 1 Bd. 8".

Gunther, l'ater in Tegernsee. Kanzelreden und Erbauungsschriften. 1 Konv. fol.

Altunünster, Kloster. Regulæe et observantixe ordinis sanctze Brigittre. P'ergumenthandschrift. 15. Jahrh. 1 tom. $4^{0}$.

Waldstetten, Kapitel. Kegula s. Augustini. Liber usuum 1673. 1 ton. 4". Freising, Domkapitel. Constitutiones et observationes Canonicorum regularium ordinis b. Angustini. 1402. Codex catenat. 4".

Regen s burg. Leichenpredigt beim Begrabniss des Bischoffen zu Regensburg, Fürsten Alberti, von Enricus Pirhing, Domprediger. 19. April 1649. 1 Heft $4^{0}$.

Freising, Hochstift. Martyrologium. Pergamenthandschrift. 10. Jahrh $1 \mathrm{Bd}$. $4^{\circ} .262 \mathrm{~s}$.

- Bibelsprüche. Pergamentrolle. 16. Jahrh. Mit Initialen.

Poesio und Dramatik.

Benedictbeuren, kloster. Gedichte, Theaterstücke, Fastnachtsspiele des Klosters. 18. Jahrh. 1 Conv.

Wernsdorf, Carl Leop. v. Politische Schwänke und Gedichte in verschie denen Dialekten und Sprachen, meist polit. Inhalts. 17. u. 18. Jahrh. 1 tom. fol.

- Politische Schwłnke und Gedichte v. J. 1614-1658. 1 Lib. fol.

Michelfeld, Kloster. Latein. Schauspiele an den Salzburger und Freisinger Gymnasien aufgefóhrt. 17. und 18. Jahrh. 4 tom. 4" und fol.

Sturm, J. Gedicht über Regensburg. Handsclirift des 18. Jahrh. 1 Bd. fol., beigebunden zu Nr. $122^{\mathrm{d}}$.

- Ein Spruch wie Herzog Albrecht von Bayern Regensburg eingenommen hat. î: 1486. 1 Lib. fol. 15. Jahrh.

Crusius. Spruchgedicht Vom kaiserlichen und Freyenstifft zu Lynda. 1548. Papierhandschrift. $2^{1 / 2}$ Bg. fol.

Gravinus, Andr. Gedichte, gedruckt und geschrieben. 1621.

Ka ste l, Klostèr. Reim-Chronik aber das Kloster v. J. 1527. 1 Lib. gr. 4".

Waldsassen, Kloster. Carmen elegiarum de miserabili dicti monasterii excidio 1504. 1 Lib. $4^{0}$.

Kaisheim, Kloster. Fragmente des mittelhochdeutschen Gedichts Wigamur. 13 Pergamentstucke. 8". 14. Jahrh.

Freising, St. Andre. Fragment de fide christiana, lat. Hexameter. Pergamenthandschrift des 12. Jahrh. nit Federzeichnungen.

Johann, Gottfried. Lobgedicht auf die Stadt Auberg mit Reihenfolge der Vorsteher der letzteren, von 1456-1614. 17. Jahrh. 1 Bd. fol.

Bartscherer, J G. Trauerlied aber den Hintritt des Churfarsten Max Emunanuel 1726. 1 Bg. $4^{0}$. 
Aus der sog. Manuskripten-Sammlung des k. allgem. Reichsarchivs. 237

Bartschererr, J. G. Gedichte, Pasquilles, Psalmen und Kriegslieder v. J. 1546. 1 Bd. 4". Ohne Decken.

Pförring, Markt. Die Schule der Handwerker, Lustspiel. Aufgeführt daselbst 1779. 1 Lib. fol.

Nagel, Anton. Literar. Nachlass. Gedichte, Lieder. 2 Fasz. fol. und 4".

- Literar. Nachlass. Gedichte, den Söhnen dę Pfalzgrafen Philipp Ludwig 1596 gewidmet.

Burgholzer, Jos Literar. Nachlass Die deutsche Henriade, ein originelles Heldengedicht in 24 Gesiingen. 1 Konv. 4"

- Literar. Nachlass. Die Psalmen Davids in deutschen Hexametern. 1 Konv. fol.

- Lutherisches Gedicht aber die Kirchenreformation im Stift Bamberg 1611. 1 Heft. $4^{0}$.

Musik-und Gesanglehre.

Latein. Choralbuch Papierhandschrift 15. Jahrh 1 tom. fol.

Deutsches Liederbuch mit Musiknoten. Papierhandschrift. Iti. Jahrh. 1 tom Querquart.

Gesangheft mit Tenornoten und deutschem Text 16. Jahrh. 1 Lib. kl. $4^{0}$. Pergamentdecke.

Is at ein. Choralbuch. Papierhandschrift. 15. Jahrh. 1 tom. fol. $230 \mathrm{~S}$.

Notenheft, Volkslieder enthaltend v. J. 1717. $1 \mathrm{Lib}$.

Choralbuch aus dem Kloster Nieder.Viehpach. Perganentcodex des 14. und 15. Jahrh. 1 tom. $4^{0}$. $130 \mathrm{Bl}$.

Martyrologium, Kirchengesănge und Litaneien mit Noten. Pergamenthandschrift. 10. Jahrh. 1 Bd. $4^{0} .262 \mathrm{~S}$.

- (Die Fortsetzung: „Alte Kopial- und Registratnrbacher im k. Reichsarchive“, folgt.) 